\title{
Paper crystals of molecular hydrogen and ice
}

\author{
The complexity of solid structures containing hydrogen bonds should be a salutary reminder that there is a long \\ way to go before some of the simplest solid structures are understood.
}

IT is not surprising that the simplest materials, bulk water and molecular hydrogen in particular, are among those whose bulk structures are the most perplexing: the mass of the hydrogen atom is only a third of that of the next heavier atom capable of forming molecules with ease. And even lithium can be a nuisance, as the long saga of attempts to calculate the structure of molecular lithium hydride attests. (Is it a molecule, or a complicated kind of atom?) Low mass, of course, implies both mobility in the classical sense and also the need to take account of the quantum properties of protons, as in the ubiquitous hydrogen bond.

Yet the arrangement of molecules in the bulk structures of these materials is of great practical importance. Liquid water is the most common of all solvents, but its versatility in still better explained by handwaving than by calculation. Molecular hydrogen is a different kettle of fish. If hydrogen is ever widely used as a transportable fuel, perhaps as a substitute for petrol or gasoline, there will no doubt be an urgent need for an accurate description of its equation of state in a form that automobile engineers can use. Meanwhile, the equation of state of pure hydrogen at pressures greater than those likely ever to be allowed on the public highways is of great concern to those who worry about the internal constitution of stars and even planets.

It is not really such a long time ago, a mere 40 years, since the late W. H. Ramsey set then-infant planetary science back on its heels with the opinion that the crude data on the mass and density of the planets could best be explained if the material at their core were hydrogen converted into metallic form (necessary to account for the Earth's magnetism) by pressure. In the event, the entertaining theory was undermined by the accumulation of better data, and has since been made entirely untenable by calculations of the transition of hydrogen to the metallic state. But who knows what happens at the centre of Jupiter or Uranus?

The problem with molecular hydrogen is easily appreciated. Suppose you arrange that hydrogen molecules are laid end to end along a line in such a way that the spacing between one end and the next is equal to the intramolecular spacing of the two protons. The result is a one-dimensional lattice with a proton on every site. The structure thus formed, by whatever magic, will not be a mere assemblage of molecules, but a unified structure in which the electrons are distributed over the entire lattice, which becomes possessed of a well-defined band structure. By promotion of electrons from the energetically lowest (and full) band, the material would behave as a semiconductor. The structure could alternatively be described as an infinite array of hydrogen molecules at least partly cemented by mutual hydrogen bonds.

The guess prompted by that simpleminded argument is that, if molecular hydrogen is compressed, long before the average distance between molecules approaches the intramolecular separation of the protons $(1.44$ atomic units, say $0.07 \mathrm{~nm}$ ), the formation of hydrogen-bonded structures will bring about departures from the behaviour of a perfect gas. In liquid molecular hydrogen, the same phenomenon should play a still more dominant role. Yet it is also known that molecular hydrogen solidifies at $14 \mathrm{~K}$, forming a hexagonally close-packed molecular solid in which molecules are separated by 3.13 a.u. That solid phase remains intact up to a pressure of $38 \mathrm{GPa}$, under which conditions the volume of the solid phase is reduced by a factor of more than five.

But what happens under more extreme conditions? Nobody is sure, which is why D. Hohl (from the Institute of Solid State Physics at Jülich) and V. Natoli, D. M. Ceperley and R. M. Martin (from the University of Illinois at Urbana-Champaign) have carried out a fiendishly complicated molecular dynamics study of the high-pressure regime (Phys. Rev. Lett. 71, 541; 1993). In doing so, they go the whole hog, treating the protons and electrons in the structure as quantum objects. To make the computation feasible, they have supposed that their bulk material is built by the repetition of basic cubes each containing 64 hydrogen atoms. The densities at which the simulations have been run correspond roughly to pressures of 35,150 and $300 \mathrm{GPa}$.

The fact that the study shows that decreasing molecular value induces the expected formation of linear chains of hydrogen molecules does not detract from its interest and importance. Because molecular dynamics allows snapshots of the molecular configuration to be extracted, it is possible to confirm that zigzag chains of hydrogenbonded atoms are formed and then are arranged in planes as the pressure is increased. But the surprise is that, even at $300 \mathrm{GPa}$, the molecular structure of what is then a solid material somehow persists. The authors promise a more detailed account of their calculations which should throw light on how the electrons behave.

The same problems arise with water in its solid phases, but more tangibly. The structure of ice has been exhaustively studied by crytallographers for half a century. (The late Dame Kathleen Lonsdale, at the Royal Institution in London towards the end of the Second World War, used to make her measurements on ice in January and February, hoping to preserve her crystal intact by keeping the laboratory window open, to the great discomfort of those around her.) Now, no fewer than a dozen solid forms of ice are known. The phase diagram is reasonably well understood, but hydrogen bonding is again the enemy of tidiness; many of these solids are inherently disordered structures except at the lowest temperature. Whether the versatility of hydrogen bonding accounts for the multiplicity of phases seems to be an open question.

The conceptual difficulty presented by a complicated phase diagram is that of understanding how one phase is derived from another by the displacement of atoms in the first structure by definite amounts. Three authors from the University of Amiens, V.P. Dmitriev, S. B. Rochal and P. Toledano (the first two "on leave" from the University of Rostov in Russia) have now tackled that formidable problem for the various forms of ice (Phys. Rev. Lett. 71, 553; 1993). One of the obvious difficulties in several of the low-pressure forms of ice is that crystalline disorder involves not merely the flipping of the allegiance of hydrogen atoms from one oxygen to another, but the presence of lattice sites that could be occupied by oxygens, but which often are not.

What Dmitriev and his colleagues have managed is to identify a body-centred substructure in unit cell of hexagonal ice (the form stable at ordinary pressure, and from which snowflakes are formed), and to show that at least nine of the 12 known forms of ice can be derived from this by specified coordinated displacement of the atoms. It is an intricate exercise of the reader's threedimensional imagination. At least one of the forms of ice not included in the analysis (ice $\mathrm{X}$ ) is understandably left out; its structure has not yet been determined.

The interest of this exercise is that, by showing that there are relatively simple ways of turning one phase into another next to it in the phase diagram, that model is made the more convincing. But in the process, the question of how the ordering of the oxygens and of the hydrogen bonds in these structures is at the same time made more clamant. That is where the theoreticians of ice structures should turn next.

John Maddox 